\title{
Original
}

\section{Morphological and Histological Changes in the Mandibular Condyle after Plate Removal in Experimental Models of Short- and Long-term Mandibular Lateral Displacement}

\author{
Jun Ando'), Masaru Yamaguchi' ${ }^{2)}$, Shouji Fujita' ${ }^{2)}$, Aya Kawamura ${ }^{2)}$, \\ Tadahiko Utsunomiya ${ }^{3)}$ and Kazutaka Kasai ${ }^{2)}$ \\ ${ }^{1)}$ Nihon University Graduate School of Dentistry at Matsudo, Orthodontics, Chiba, Japan \\ ${ }^{2}$ Departments of Orthodontics, Nihon University School of Dentistry at Matsudo, Chiba, Japan \\ ${ }^{3}$ Departments of Oral Pathology, Nihon University School of Dentistry at Matsudo, Chiba, Japan \\ (Accepted for publication, January 8, 2013)

\begin{abstract}
We conducted an in vivo study to clarify the mechanics of the mandibular bone and condyles in pediatric patients with mandibular functional deviation. We experimentally caused lateral displacement of the mandible in 4-week-old rats by attaching a plate to the teeth for 4 (short-term group: ST group) or 12 weeks (long-term group: LT group), and immunohistochemically investigated the pattern of appearance of matrix metalloproteinase-1 (MMP-1), vascular endothelial growth factor (VEGF), and connective tissue growth factor (CTGF) to investigate the changes in the cartilage matrix constitution during the recovery of the condylar morphology. Furthermore, the morphology was observed using micro $(\mu)-\mathrm{CT}$.

After plate removal, the MMP-1 expression significantly decreased in the both groups. Furthermore the expressions of VEGF and CTGF were increased in the ST group compared to those observed in the LT group. In terms of the morphometry, mandibular asymmetry and deformity of the condyle were noted immediately after removal in the both groups. The mandibular morphology markedly recovered in the ST group compared to that in the LT group. This indicates that correction of occlusal abnormalities during an early stage leads to recovery of the normal mandibular configuration.
\end{abstract}

Key words: Condyle, Type X collagen, VEGF, CTGF, Asymmetry

\section{Introduction}

The pathogenesis of jaw deformity has not yet been fully elucidated, although the causes are likely to be multifactorial ${ }^{1,2}$, and malocclusion is also considered to be one of the contributors ${ }^{3)}$. Generally, malocclusion and facial malformation occur due to inhibition of normal development, not arising from a specific morbid process. It has been speculated that the malocclusion induced by functional lateral displacement of the lower jaw during periods of growth is likely to gradually lead to skeletal displacement ${ }^{4}$. In such cases, the jaw bone can be modified to grow normally by improving the functional cause at an early stage and minimizing the deformity ${ }^{5,6}$. In order to clarify the mechanics of the mandibular bone and condyles in pediatric patients with mandibular functional deviation who visited the Department of Orthodontics, we performed in vivo observation of the recovery process of the functional deviation after resolving the cause of

Correspondence to: Dr Masaru Yamaguchi, Department of Orthodontics, Nihon University School of Dentistry at Matsudo, 2-870-1 SakaechoNishi, Matsudo, Chiba, 271-8587, Japan. Tel: +81-47-360-9414; Fax: +81-47-364-6295; E-mail: yamaguchi.masaru@nihon-u.ac.jp the deviation.

Several studies of lateral displacement of the mandible have investigated changes of the articular condyle and mandible in experimental models, in which a side shift plate was attached to the anterior teeth to produce mandibular displacement. Nakano ${ }^{7)}$ reported that leftward displacement of the mandible results in a long mandible on the right side and a high ramus of the mandible on the left side. Fuentes et al. $\left.{ }^{8}\right)$ reported that rightward displacement of the mandible thickened the mandibular condyle on the left side in rats during the growth period, and Petrovic et al. ${ }^{9)}$ reported that compression of the mandibular condyle with a chin cap inhibited the growth of the mandibular condylar cartilage layer.

Our previous study investigated morphological and histological changes in the condyle after removing the cause of the changes in an experimental model of lateral displacement of the mandible ${ }^{10)}$. They observed that attachment of a site shift plate caused mandibular displacement, and removal of the cause induced a reduction of the production of matrix metalloproteinase (MMP)$1,-8$ and -13 and increased that of type II collagen, through which 

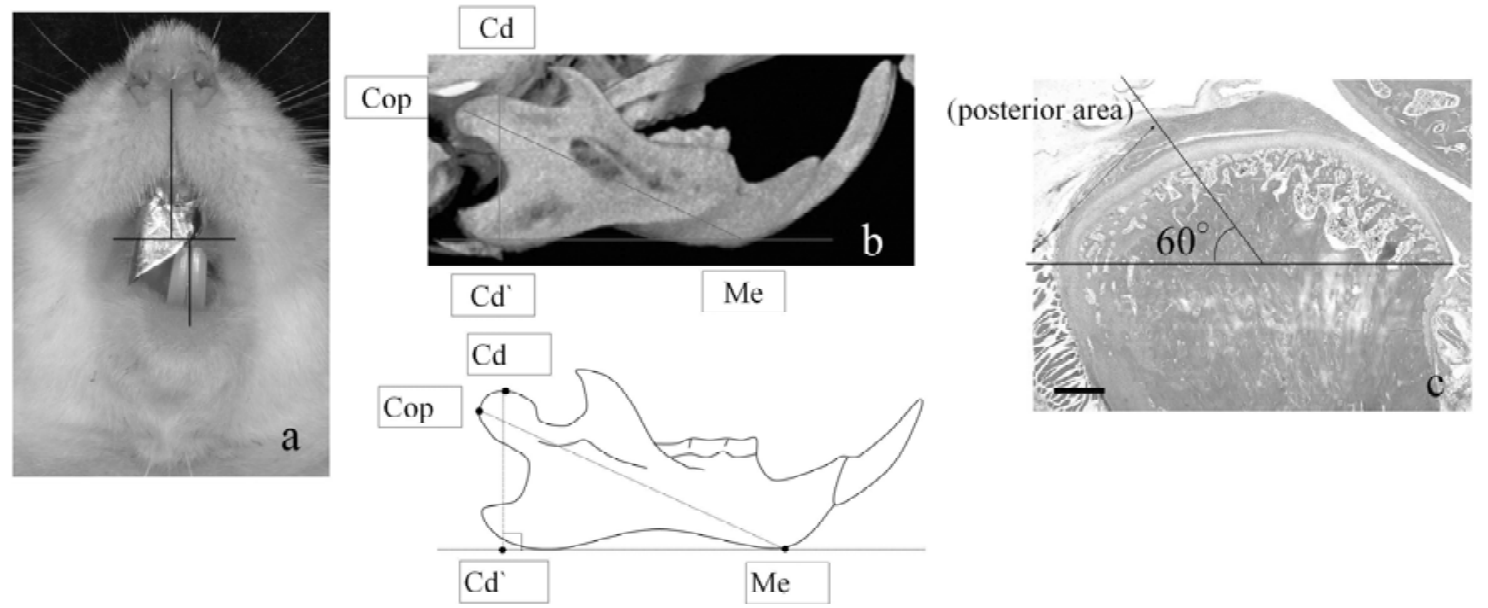

Figure 1. A side shift plate, cast using silver alloy, was attached to the upper anterior teeth to induce mandibular displacement to the left side (a). Reference points and measurement parameters for the rat mandible. Me: Menton, Cd: The point on the top of the condyle, Cop: The point of the posterior of condyle, $\mathrm{Cd}^{\prime}:$ Perpendicular to the mandibular plane from the condyle, Cd-Cd': Mandibular ramus height, Me-Cop: Mandibular length (b). The area where the levels of MMP-1, VEGF and CTGF were measured. Deeply stained portions were quantified using the Image-Pro Plus software program (version 5.0: Media Cybernetics) in the posterior area of the upper part of the articular condyle, which was split into three portions at the midpoint of the horizontal line drawn from the most prominent part of the articular condyle (bar: $500 \mu \mathrm{m})(\mathrm{c})$.
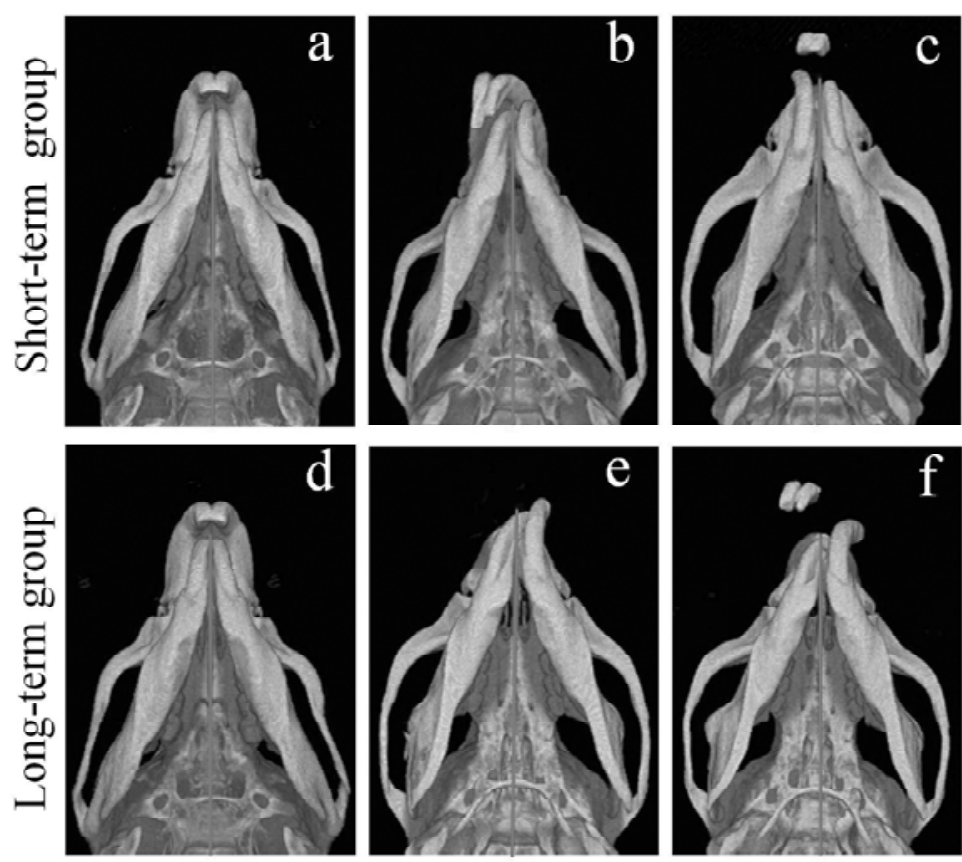

Figure 2. The mandible was imaged by $\mu$-CT. The ST group: Start (a), immediately after removal of the plate (b), 4 week after removal of the plate (c). The LT group: Start (d), immediately after removal of the plate (e), 12 week after removal of the plate (f).

condylar cartilage grew and led to the recovery of the condylar morphology ${ }^{11)}$. These findings suggested that malocclusioninduced changes in the condylar cartilage are closely involved in generating mandibular deformities. However, there has been no report in which the plate attachment period was divided into early and late periods, and where the changes were compared using rats maintained with an identical diet under identical conditions. In this study, long-term serial micro $(\mu)$-CT observation of the condylar and mandibular bone morphology of the same rat and immunostaining of the condylar cartilage were performed.

Vascular endothelial growth factor (VEGF) is produced by not only vascular endothelial cells, but also osteoblasts and enlarged chondrocytes, and is involved in bone remodeling ${ }^{12)}$. Connective tissue growth factor (CTGF) shows diverse functions in the proliferation and differentiation of chondrocytes, osteoblasts, fibroblasts, and vascular endothelial cells. However, the changes in VEGF and CTGF after removal of the cause of the deformity in an experimental model of lateral displacement of the mandible 
Jun Ando et al.: Changes in the Mandibular Condyle

Table 1: Measurements of mandibular by $\mu-\mathrm{CT}(\mathrm{mm})$

\begin{tabular}{|c|c|c|c|c|c|c|c|}
\hline & \multicolumn{3}{|c|}{ Mandibular ramus height } & \multicolumn{4}{|c|}{ Mandibular length } \\
\hline & \multicolumn{2}{|c|}{ ST group (A) } & LT group (B) & \multicolumn{2}{|c|}{ ST group (C) } & \multicolumn{2}{|c|}{ LT group (D) } \\
\hline & ipsilateral side & contralateral side & ipsilateral side contralateral side & ipsilateral side & contralateral side & ipsilateral side & contralateral side \\
\hline start & $7.66 \pm 0.23$ & $7.62 \pm 0.25$ & $7.87 \pm 0.23$ & $15.50 \pm 0.31$ & $15.53 \pm 0.24$ & $15.46 \pm 0.35$ & $15.48 \pm 0.24$ \\
\hline 0 & $9.16 \pm 0.24$ & $9.22 \pm 0.27$ & $9.92 \pm 0.22 * * \overline{9.36} \pm 0.36$ & $17.49 \pm 0.24$ & $\overrightarrow{18.11} \pm 0.28$ & $18.88 \pm 0.21$ & $\widehat{19.81} \pm 0.26$ \\
\hline 1 & $9.76 \pm 0.24$ & $9.82 \pm 0.21$ & $10.01 \pm 0.26 * \overrightarrow{9.45 \pm 0.21}$ & $17.81 \pm 0.23$ & $\overrightarrow{18.33} \pm 0.32$ & $19.06 \pm 0.23$ & $\widehat{19.79} \pm 0.27$ \\
\hline 2 & $9.86 \pm 0.16$ & $9.87 \pm 0.43$ & $* \frac{1}{9.55 \pm 0.34}$ & $18.74 \pm 0.42$ & $19.13 \pm 0.31$ & $19.30 \pm 0.25 *$ & $\overline{19.98} \pm 0.24$ \\
\hline 4 & $10.25 \pm 0.24$ & $10.18 \pm 0.21$ & $10.43 \pm 0.21 * 2.96 \pm 0.23$ & $19.28 \pm 0.25$ & $19.18 \pm 0.24$ & $19.66 \pm 0.3$ & $20.35 \pm 0.26$ \\
\hline 8 & $10.66 \pm 0.33$ & $10.78 \pm 0.15$ & 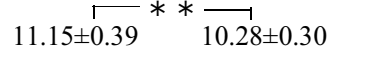 & $19.95 \pm 0.27$ & $19.89 \pm 0.27$ & $20.47 \pm 0.28$ & $\overline{21.13} \pm 0.31$ \\
\hline 12 & $10.98 \pm 0.21$ & $10.95 \pm 0.23$ & $11.20 \pm 0.33 * * \overline{10.49 \pm 0.21}$ & $21.42 \pm 0.23$ & $21.28 \pm 0.39$ & $20.87 \pm 0.35 *$ & $\overrightarrow{21.68} 8 \pm 0.32$ \\
\hline
\end{tabular}

The mandibular ramus height was measured perpendicular to the mandibular plane from the condyle. The ST group (A): There were no significant differences between the ipsilateral (Left) and contralateral sides (Right). The LT group (B): There were significant differences compared to the corresponding contralateral side. ( $*: \mathrm{p}<0.05, * *: \mathrm{p}<0.01$, by one-way ANOVA)

The mandibular length was measured from condyle to menton. The ST group (C): The length was significantly different from the corresponding the contralateral side. $(*: p<0.05$, by one-way ANOVA). The LT group (D): There were significant differences compared to the corresponding contralateral side. $(*: \mathrm{p}<0.05, * *: \mathrm{p}<0.01$, by one-way ANOVA)

Table 2: Area of VEGF $\left(\mu \mathrm{m}^{2}\right)$

\begin{tabular}{|c|c|c|c|c|c|c|}
\hline \multirow[b]{3}{*}{ week } & \multicolumn{6}{|c|}{ VEGF } \\
\hline & \multicolumn{3}{|c|}{ ST group } & \multicolumn{3}{|c|}{ LT group } \\
\hline & control & ipsilateral side & contralateral side & control & ipsilateral side & contralateral side \\
\hline 0 & $30582.3 \pm 2865.2$ & $69917.3 \pm 4352.3^{++}$ & $60367.0 \pm 5946.0 * *$ & $30106.3 \pm 5569.3$ & $50372.3 \pm 5172.3^{++}$ & $54861.7 \pm 5786.9^{* *}$ \\
\hline 1 & $35052.0 \pm 4500.0$ & $74921.7 \pm 3835.6^{++}$ & $57225.3 \pm 5023.3 * *$ & $26373.3 \pm 2471.6$ & $68191.7 \pm 4209.2^{++}$ & $60541.7 \pm 8043.5 * *$ \\
\hline 2 & $39952.0 \pm 1983.3$ & $45118.3 \pm 4005.5$ & $65289.3 \pm 5371.1 * *$ & $34676.3 \pm 3598.2$ & $34703.0 \pm 3775.1$ & $55403.7 \pm 5026.2 * *$ \\
\hline 4 & $40195.3 \pm 6103.6$ & $30048.0 \pm 5982.6$ & $40295.7 \pm 8054.8$ & $29830.7 \pm 4464.3$ & $40063.3 \pm 3442.9$ & $39982.3 \pm 5982.3$ \\
\hline 8 & $39664.7 \pm 7542.2$ & $38134.0 \pm 2664.4$ & $38322.0 \pm 3007.5$ & $30006.3 \pm 2644.7$ & $35917.7 \pm 4110.3$ & $35216.7 \pm 4226.6$ \\
\hline
\end{tabular}

Vertical axis of the graph represents weeks after removal of the side shift plate (week).

The asterisk $(*)$ indicates a significant difference between the contralateral (Right) side and control.

The plus sign $(+)$ indicates a significant difference between the ipsilateral (Left) side and control. $\left({ }^{* *}: \mathrm{p}<0.01,++\right.$ :p $<0.01$, by one-way ANOVA)

have not been sufficiently elucidated.

In this study, we experimentally caused lateral displacement of the mandible by attaching a side shift plate to the anterior teeth of the maxilla in male four-week-old rats, in which endochondral ossification is active, for 4 or 12 weeks, and immunohistologically and immunohistochemically investigated the pattern of appearance of MMP-1, VEGF and CTGF to investigate the changes in the cartilage matrix constitution during the recovery of the condylar morphology after removal of the cause of the displacement.

\section{Materials and Methods}

\section{Experimental animals}

Ninety-nine 4-week-old male Wistar rats were used: 11 animals for observation of the morphological changes in the mandible and condyle and 88 animals for the histopathological and immunohistochemical examinations. The animals were randomly divided into control and experimental groups comprising 3 and 4 animals, respectively, for observation of the morphological changes. Additionally, the animals were divided into control and experimental groups of 4 animals each for the histopathological and immunohistochemical examinations. The animals were reared in SPF clean cages at the Experimental Animal Center of Nihon University School of Dentistry at Matsudo. The softer diet (MR Powder Diet, Sankyo Labo Service, Co., Tokyo, Japan), drinking water (tap water given ad libitum), floor mats, and cages were all sterilized before use. Both the control and experiment groups were given the same diet. All animal experiments were performed in accordance with the Ethical Guidelines for Animal Experiments of our institution (Approval No. AP11-MD024). 
J.Hard Tissue Biology Vol. 22(2):201-210, 2013

Table 3: Area of CTGF $\left(\mu \mathrm{m}^{2}\right)$

\begin{tabular}{|c|c|c|c|c|c|c|}
\hline \multirow[b]{3}{*}{ week } & \multicolumn{6}{|c|}{ CTGF } \\
\hline & \multicolumn{3}{|c|}{ ST group } & \multicolumn{3}{|c|}{ LT group } \\
\hline & control & ipsilateral side & contralateral side & control & ipsilateral side & contralateral side \\
\hline 0 & $40950.3 \pm 4209.7$ & $65447.3 \pm 5421.9^{++}$ & $60133.0 \pm 5086.7^{* *}$ & $27099.7 \pm 4997.5$ & $50381.3 \pm 4014.3^{++}$ & $50170.7 \pm 4210.1^{* *}$ \\
\hline 1 & $49520.0 \pm 4131.9$ & $80409.0 \pm 4705.6^{++}$ & $64692.0 \pm 5175.4 * *$ & $32870.7 \pm 3009.0$ & $48431.0 \pm 5164.8^{++}$ & $45521.7 \pm 6122.1^{* *}$ \\
\hline 2 & $48521.0 \pm 3208.5$ & $81175.7 \pm 5771.5^{++}$ & $60410.7 \pm 2117.6^{* *}$ & $32449.0 \pm 4043.8$ & $36628.3 \pm 4996.5$ & $52718.0 \pm 4067.1^{* *}$ \\
\hline 4 & $47226.0 \pm 2274.6$ & $55136.3 \pm 5399.6$ & $70199.3 \pm 5031.9^{* *}$ & $37458.3 \pm 3675.9$ & $33007.3 \pm 5292.6$ & $37704.7 \pm 2887.0$ \\
\hline 8 & $35152.0 \pm 2735.9$ & $40554.3 \pm 3707.0$ & $40185.0 \pm 4179.0$ & $27484.7 \pm 3178.8$ & $27767.0 \pm 2903.3$ & $25825.7 \pm 1928.3$ \\
\hline
\end{tabular}

Vertical axis of the graph represents weeks after removal of the side shift plate (week).

The asterisk $(*)$ indicates a significant difference between the contralateral (Right) side and control.

The plus sign $(+)$ indicates a significant difference between the ipsilateral (Left) side and control. $(* *: p<0.01,++:$ p $<0.01$, by one-way ANOVA)

Table 4: Area of MMP-1 $\left(\mu \mathrm{m}^{2}\right)$

\begin{tabular}{|c|c|c|c|c|c|c|}
\hline \multirow[b]{3}{*}{ week } & \multicolumn{6}{|c|}{ MMP-1 } \\
\hline & \multicolumn{3}{|c|}{ ST group } & \multicolumn{3}{|c|}{ LT group } \\
\hline & control & ipsilateral side & contralateral side & control & ipsilateral side & contralateral side \\
\hline 0 & $12944.7 \pm 1998.2$ & $30232.0 \pm 1957.4^{++}$ & $20805.0 \pm 2193.4^{* *}$ & $11024.0 \pm 585.4$ & $26258.0 \pm 1879.5^{++}$ & $20080.0 \pm 1988.5^{* *}$ \\
\hline 1 & $14228.0 \pm 1471.2$ & $18417.3 \pm 464.6^{++}$ & $20186.0 \pm 2291.3^{* *}$ & $12247.7 \pm 1227.7$ & $16441.3 \pm 1428.8^{++}$ & $18059.6 \pm 1507.2^{* *}$ \\
\hline 2 & $14590.0 \pm 1014.0$ & $16364.0 \pm 1257.9$ & $15417.7 \pm 1718.5$ & $12811.3 \pm 958.9$ & $12342.7 \pm 1991.7$ & $13092.0 \pm 1227.2$ \\
\hline 4 & $12244.0 \pm 1364.6$ & $13330.7 \pm 1344.5$ & $12145.7 \pm 1851.0$ & $12046.7 \pm 1151.6$ & $13023.3 \pm 1708.9$ & $12036.3 \pm 1535.5$ \\
\hline 8 & $12253.7 \pm 839.5$ & $13030.3 \pm 839.9$ & $12814.0 \pm 1661.7$ & $11020.3 \pm 930.0$ & $10884.3 \pm 190.1$ & $11262.3 \pm 2093.9$ \\
\hline
\end{tabular}

Vertical axis of the graph represents weeks after removal of the side shift plate (week).

The asterisk $(*)$ indicates a significant difference between the contralateral (Right) side and control.

The plus sign $(+)$ indicates a significant difference between the ipsilateral (Left) side and control. $(* *$ :p $<0.01,++$ :p $<0.01$, by one-way ANOVA)

\section{Animal treatment and maintenance conditions}

The rats were anaesthetized with pentobarbital sodium (40 $\mathrm{mg} / \mathrm{kg}$ body weight) for the application of side shift plates. An anesthetic was given in the pathologic section preparation group and the $\mathrm{CT}$ radiography control and experimental groups. A side shift plate, cast using silver alloy, was attached to the upper anterior teeth (Fig. 1a) to induce mandibular displacement to the left side, and then was removed after 4 or 12 weeks. Plate attachment period group of 4 and 12 weeks were assumed each a short term group (ST group) and long term group (LT group). The side shift plate was attached using Super Bond adhesive (San Medical, Co., Shiga, Japan) after treating all areas of the upper erupted incisor with $65 \%$ phosphate for $60 \mathrm{~s}$. Histopathological and immunohistochemical examinations were performed at $0,1,2,4$ and 8 weeks after removal of the device. The left side was the ipsilateral side and the right was the contralateral side in all of the experimental rats. Morphological changes were observed at $0,1,2,4,8$ and 12 weeks after removal of the device.

\section{Morphometric items}

The body weight was measured weekly to confirm the whole body growth of the rats. The mandible was imaged at $0,1,2,4,8$ and 12 weeks after plate attachment. The acquisition conditions were: magnification power, 2.0 ; tube voltage, $90 \mathrm{kV}$; tube current, $50 \mu \mathrm{A}$; and acquisition time, $17 \mathrm{sec}$. The acquired images were reconstructed into three-dimensional (3-D) images using 3-D construction software (TRI/3D-BMD, RATOC, Co., Tokyo, Japan), and the items shown in Fig. 1b were measured employing a modification of the methods by Watanabe et al. ${ }^{10)}$ and Nakano ${ }^{7)}$. Furthermore, we established planes so that the mandibular plane became parallel to the floor (XZ plane) and the frontal section of the maxillary bone became perpendicular to the mandibular plane.

\section{Preparation of specimens}

According to the experimental schedule, the animals were placed under anesthesia, then about $250 \mathrm{ml}$ of a normal saline solution was injected into the left ventricle for avascularization. Perfusion fixation was performed using a $10 \%$ neutral formalin solution, and the bilateral articular condyles were extracted and re-fixed in a $10 \%$ neutral formalin solution at $4{ }^{\circ} \mathrm{C}$ for one week. Following fixation, the samples were decalcified in a $10 \%$ disodium ethylenediamine tetracetic acid (EDTA, pH 7.4) at room 
Jun Ando et al.: Changes in the Mandibular Condyle

temperature for three weeks. The decalcified samples were rinsed under running water and embedded in paraffin to prepare a block using a standard method. Sagittal sections of the condyle samples were sliced at $4 \mu \mathrm{m}$ thick and stained using various techniques.

For immunohistochemical staining, Histofine Simple Stain Rat MAX-PO (R, G) kit (Nichirei, Co., Tokyo, Japan) was used. As the primary antibodies, anti-rabbit matrix metalloproteinase-1 polyclonal antibody (Santa Cruz Biotechnology, Co., Santa Cruz, CA, USA; dilution ratio 1:40), anti-goat VEGF antibody (R\&D SYSTEMS, Co., Minneapolis, MN, USA; dilution ratio 1:20), and an anti-goat CTGF polyclonal antibody (Santa Cruz Biotechnology, Co., Santa Cruz, CA, USA; dilution ratio 1:50) were used.

After deparaffinization and rinsing with TBS, each section was left to react for 15 minutes in $3 \% \mathrm{H}_{2} \mathrm{O}_{2}$ in methanol, then reactions were performed with the above-mentioned primary antibodies overnight at $4{ }^{\circ} \mathrm{C}$. After the antibody reactions, the sections were subjected to reactions with the Histofine Simple Stain Rat MAX-PO $(\mathrm{R}, \mathrm{G})$ at room temperature for 30 minutes. After developing the samples in DAB solution, they were counterstained using Mayer's hematoxylin solution.

\section{Histopathological observations}

Tissue sections from each group were stained with hematoxylineosin (H-E) and were subjected to immunohistochemical staining. The sections were observed under a light microscope. Histopathological observations of the articular condyle were categorized in accordance with Kameyama's classification ${ }^{13)}$, as follows: The cartilage was classified into five layers, namely, a layer of fibrous connective tissue, a cartilage layer of proliferating cells (layer of proliferation), a cartilage layer of differentiated cells (layer of differentiation), a cartilage layer of hypertrophic cells (layer of hypertrophy), and a layer of ossification.

\section{Determination of areas stained for MMP-1,VEGF and CTGF}

Areas of hot-spot reactions in the posterior area of the upper condyle were split into three portions at the midpoint of the horizontal line drawn from the most prominent point of the articular condyle as reported by Watanabe et al. ${ }^{11)}$ and were measured using the Image-Pro Plus software program (version 5.0: Media Cybernetics) (Fig. 1c).

\section{Statistical methods}

Data were analyzed for statistically significant differences using a one-way analysis of variance (one-way ANOVA), followed by multiple comparisons with Scheffe's F test (Tables 1-4). A comparison was made between Right and Left in Table1 and between Right/Left and Control in Tables 2-4.

\section{Results}

\section{Body weight}

In the ST group, the body weight increased similarly to that in the control group, but a difference was noted between the body weights after 1 to 2 weeks of side shift plate attachment and at 1 to 2 weeks after removal of the plate. However, no significant difference was noted between the control and experimental groups at the completion of the experiment.

In the LT group, the body weight increased similarly to that in the control group, but a difference was noted between the body weights after 1 to 3 weeks of side shift plate attachment and at 1 to 2 weeks after removal of the plate. As in the ST group, no significant difference was noted between the control and experimental groups at the completion of the experiment (date not shown).

\section{Morphometry of the mandible and condyle Changes in the mandible}

Macroscopically, mandibular asymmetry was noted immediately after removal of the plate in the ST and LT groups. Furthermore, in both groups, the mandibular anterior teeth were deviated by approximately $1.5 \mathrm{~mm}$ on average compared with the maxillary anterior teeth (Fig. 2-b, e). In the ST group, no mandibular asymmetry was noted beginning 4 weeks after removal (Fig. 2-c). Meanwhile, in the LT group, mandibular asymmetry was also noted 12 weeks after removal (Fig. 2-f).

\section{Changes in the condyle}

The posterior region of the condyle on the ipsilateral side was observed to be flattened immediately after removal of the plate in the ST group (Fig. 3-b). Later, the condyle returned to the same state as that observed at the time of the initiation of the experiment at 4 weeks after removal (Fig. 3-c). In the LT group, the posterior region of the condyle on the displaced side was noted to be flattened immediately after plate removal (Fig. 3-e), and the condyle had not returned by 12 weeks after removal (Fig. 3-f).

\section{Mandibular ramus height}

In the ST group, no significant difference was noted in the mandibular ramus height between the ipsilateral and contralateral sides at any time point (Table 1-A). In contrast, in the LT group, the mandibular ramus height was significantly greater on the ipsilateral than on the contralateral side at all time points (Table 1-B).

\section{Mandibular length}

In the ST group, the mandibular length was significantly smaller on the ipsilateral side than on the contralateral side immediately and 1 week after plate removal, but no significant difference was noted at 2, 4, 8 and 12 weeks after removal (Table 1-C). In the LT group, the length was significantly greater on the contralateral side than on the ipsilateral side at all time points (Table 1-D). 

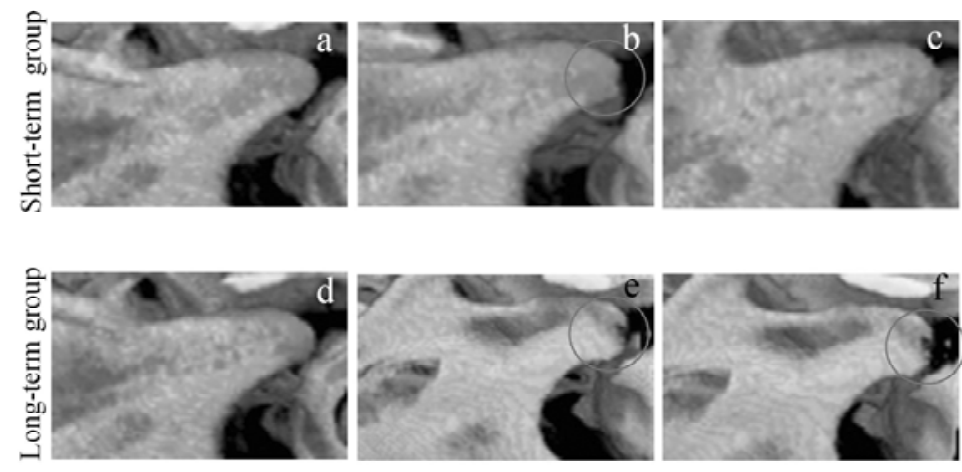

Figure 3. Changes in the condyle (ipsilateral side, left side) were observed by $\mu-C T$. The ST group: Start (a), immediately after removal of the plate (b), 4 weeks after removal of the plate (c). The LT group: Start (d), immediately after removal of the plate (e), 12 weeks after removal of the plate (f). The posterior region of the condyle was noted to be flattened (b, d,f).
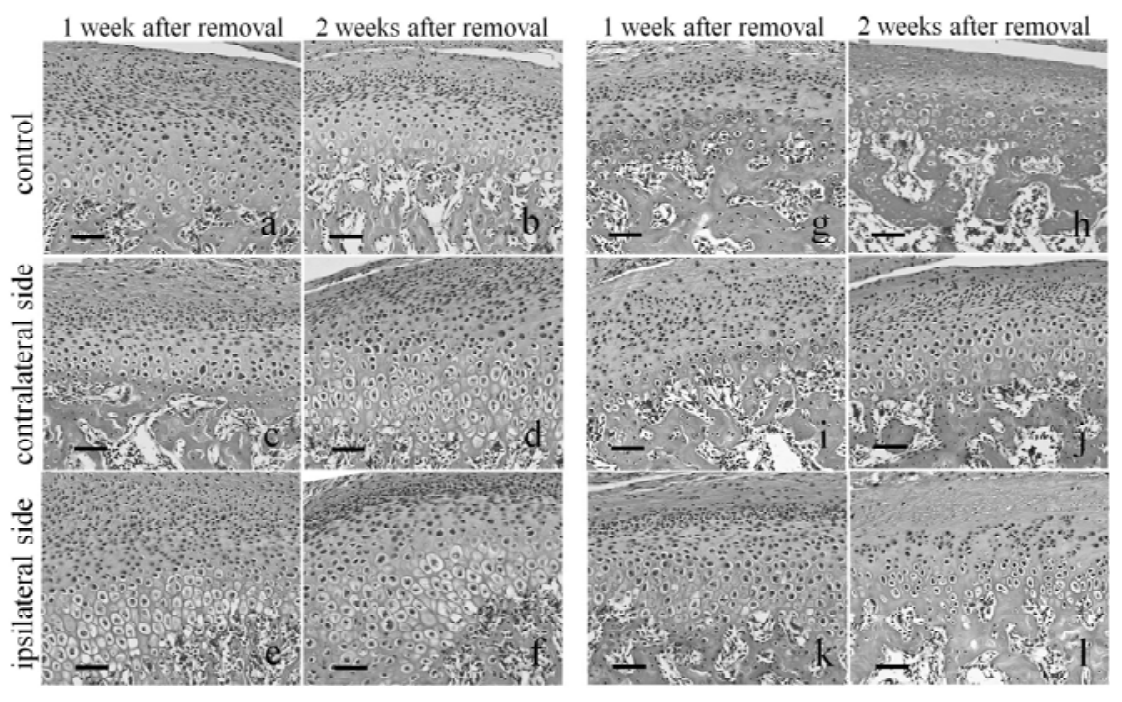

Figure 4. Histopathological findings (H-E) (bar: $50 \mu \mathrm{m}$ ).

ST group : a-f, LT group : g-l, control: a-b, g$\mathrm{h}$, contralateral side (right side): $\mathrm{c}-\mathrm{d}, \mathrm{i}-\mathrm{j}$, ipsilateral side (left side): e-f, k-1

\section{Histopathological findings (H-E staining) Control group}

In the ST group, the superficial layer of the articular condyle was covered with thin laminated fibrous connective tissue. Beneath the fibrous connective tissue layer, a layer of proliferative cells, in which small oval or elliptical cellular components were densely distributed, was present. Further down, in the hypertrophic layer, relatively large circular or elliptical cartilaginous cells were arranged in a bean-like pattern. Between the layers of proliferation and hypertrophy, a transitional layer of differentiation was observed. Furthermore, beneath the hypertrophic layer, new trabecular bone arranged in a complicated mosaic structure was found (Fig. 4: a-b).

Similar findings were noted in the LT group, but the proliferative, differentiating, and hypertrophic layers were decreased in the LT group compared to those in the ST group (Fig. 4: g-h).

\section{Experimental group}

Loss of the hypertrophic layer and invagination of this layer into the proliferative layer were noted on the ipsilateral side immediately after removal of the side shift plate in the ST group. One week after the removal, the cartilage layer (mainly thickening of the layers of proliferation and hypertrophy) had proliferated and new trabecular bone was observed. Eight weeks after removal, findings similar to those of the control group were observed. With regard to the contralateral side, 2 weeks after removal, the cartilage layer (mainly thickening of the layers of proliferation and hypertrophy) had proliferated. The timing of proliferation of the layer of hypertrophy varied between the sides (Fig. 4: c-f). Similar findings were noted in the LT group (Fig. 4: i-1).

\section{VEGF staining \\ Control group}

In the ST and LT groups, positive reactions were observed in the territorial matrix of the proliferative, differentiating, and hypertrophic chondrocyte layers when the experiment was initiated. Subsequently, positive findings were identified in the cellular matrix of the layers of differentiating and hypertrophic cells throughout the experimental period (Fig. 5: a-b, g-h).

\section{Experimental group}

In the ST and LT groups, positive reactions were observed in the territorial matrix in the proliferative and differentiation layers on the ipsilateral and contralateral sides immediately after the removal of the side shift plate (at the time of plate removal). At 1, 
Jun Ando et al.: Changes in the Mandibular Condyle
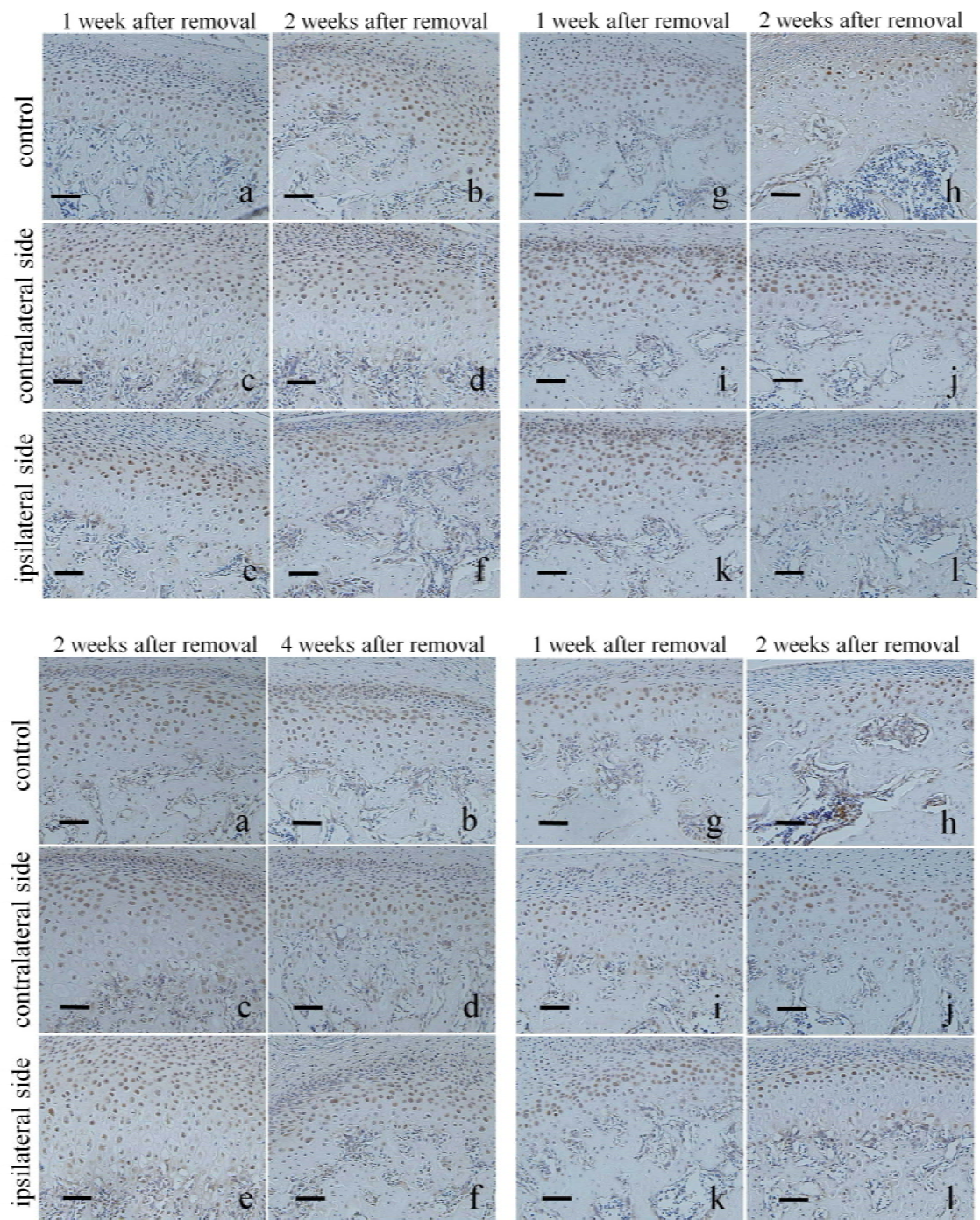

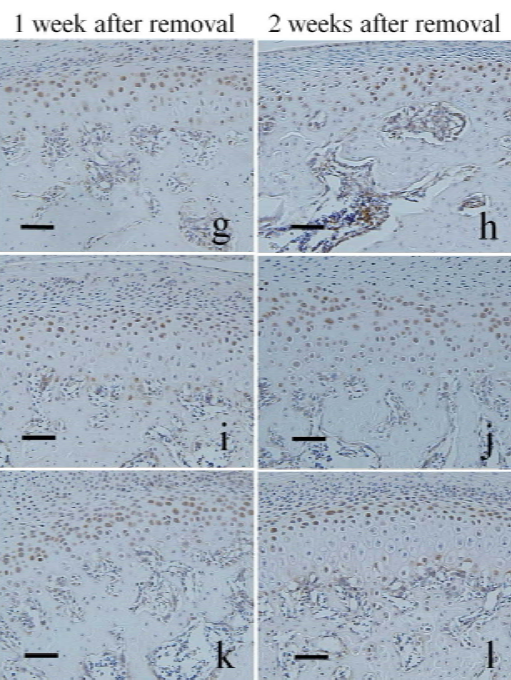

Figure 5. Immunohistochemical findings of VEGF (bar: $50 \mu \mathrm{m}$ ).

ST group : a-f, LT group : g-l, control: a-b, gh, contralateral side (right side): $c-d, i-j$, ipsilateral side (left side): e-f, k-1

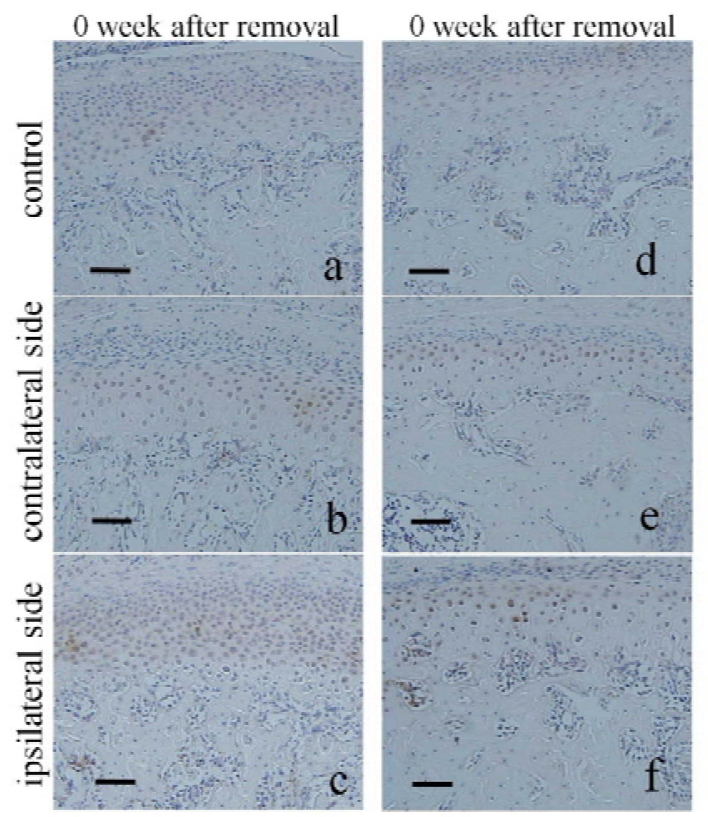

Figure 7. Immunohistochemical findings of MMP-1 (bar: $50 \mu \mathrm{m}$ ) ST group : a-c, LT group : d-f, control: a,d, contralateral side (right side): b,e, ipsilateral side (left side): $c, f$
2, 4 and 8 weeks after removal, positive findings were observed in the cellular area matrix of the layers of differentiation and hypertrophy (Fig. 5: c-f, i-1). On the ipsilateral sides 1 week after removal and contralateral side 2 weeks after removal, a significant increase was observed, while the size of the area declined thereafter (Table 2). A comparison of the results was made between Right/ Left and control.

\section{CTGF staining Control group}

In both the ST and LT groups, positive reactions were observed in the territorial matrix in the proliferative and differentiation layers and in part of the hypertrophic chondrocyte layer at the beginning of the experiment. Subsequently, positive findings were identified in the cellular area matrix of the layer of differentiation and a part of the hypertrophic layer throughout the experimental period (Fig.6: a-b, g-h).

\section{Experimental group}

In the ST and LT groups, positive reactions were observed in 
the territorial matrix in the proliferative and differentiation layers on both the ipsilateral and contralateral sides immediately after plate removal (at the time of plate removal). At 1, 2, 4 and 8 weeks after removal of the plate, positive findings were observed in the cellular area matrix of the layers of differentiation and in part of the hypertrophic layer (Fig. 6: c-f, i-l). The CTGF-stained areas significantly increased on the ipsilateral and contralateral sides from immediately to 1 week after plate removal in both the ST and LT groups, while the size of the area declined thereafter. When the area after plate removal was compared between the ST and LT groups, the area tended to have decreased more in the LT group (Table 3). A comparison of the results was made between Right/ Left and control.

\section{MMP-1 staining \\ Control group}

In the ST and LT groups, slightly positive findings for MMP1 were observed in the extracellular matrix of the layer of proliferation and cellular area matrix of the layer of differentiation throughout the experimental period (Fig. 7: a,d).

\section{Experimental group}

In the ST and LT groups, positive reactions were observed in the territorial matrix in the proliferative and differentiated layers on both the ipsilateral and contralateral sides immediately after plate removal (Fig. 7:b-c, e-f). Subsequently, the levels of staining declined. At 8 weeks after removal of the plate, findings similar to those of the control group were found. For the areas examined on both the ipsilateral and contralateral sides, a significant increase was identified compared to the area in the control group immediately after removal of the plate, while the size of the areas decreased thereafter. No significant difference in the area was observed between the control and experimental groups beginning 2 weeks after removal on the contralateral side and from 1 week after removal on the ipsilateral side (Table 4).

\section{Discussion}

In the present study, we used a side shift plate attached to the maxilla, as reported by Watanabe et al. ${ }^{10}$, that is able to reproduce conditions similar to the jaw displacement induced by functional factors during mandibular movement. As a result, asymmetry of the mandible (Fig. 2-b, e) and resorption of the articular condyle (Fig. 3-b, e) were observed immediately after removal of the plate. Furthermore, resorption of the posterior region of the articular condyle on the ipsilateral side was noted immediately after removal of the plate. Watanabe et al. ${ }^{11)}$ also reported that lateral displacement of the mandible causes mechanical pressure on the ipsilateral side. Accordingly, this method is effective for experimentally inducing jaw deformities associated with asymmetry.
It was speculated that this loss of weight was due to the eating disorder caused by the attachment and removal of the plate. However, at the end of the experiment, no significant differences were observed between the experimental and control groups. Therefore, we considered the eating disorders to be temporary and determined that the influence of the plate on general health could be disregarded.

In terms of the morphometry, the mandibular length was significantly shorter on the ipsilateral side than on the contralateral side both immediately and 1 week after removal of the side shift plate in the ST group, but no significant difference was noted between the two sides after 2 weeks (Table 1-C). The morphology of the flattened posterior region of the mandibular condyle on the ipsilateral side returned after plate removal (Fig. 3). Nakano ${ }^{7)}$ previously reported that the mandible was significantly shorter on the experimentally ipsilateral side from 2 weeks after initiation of the experiment until completion at 37 weeks. We observed a similar finding immediately after plate removal. Watanabe et al. ${ }^{11)}$ induced mandibular displacement using a side shift plate adhering to the maxilla, and observed that compression occurred in the posterior region of the condyle on the ipsilateral side, inhibiting its growth, while the condyle recovered the original morphology or size after plate removal due to a catch-up growth phenomenon ${ }^{14)}$. A similar phenomenon may have occurred after plate removal in the ST group in the present study.

In the LT group, the mandibular length was significantly shorter on the ipsilateral side than on the contralateral side throughout the period after the side shift plate was removed. Miki ${ }^{15)}$ reported that the mandibular condyle smoothly grew until about 100 days after birth, but that the growth stopped thereafter, and it maintained a constant size, thus suggesting that recovery of the condylar morphology and growth/development, serving as the center of mandibular growth, was not active after plate removal in the LT group, while it was still active in the ST group.

Regarding the mandibular ramus height, no significant difference was noted between the two sides throughout the period after plate removal in the ST group. Watanabe et $\mathrm{al}^{10)}$ reported that growth was inhibited only in the posterior region of the mandibular condyle on the ipsilateral side due to compression, resulting in no difference in the laterality of the mandibular ramus height. Similarly, growth inhibition may have occurred in the posterior region of the mandibular condyle, causing no change in the mandibular ramus height in the ST group. In the LT group, the height was significantly greater on the ipsilateral side than on the contralateral side throughout the period after plate removal. $\mathrm{Nakano}^{7)}$ reported that the bone mineral density and bone morphology changed in the mandibular angle region after the lateral appearance of masseter tissue, and the changes in the masseter tissue, bone mineral density, and bone morphology started at 9,12 and 15 weeks of age, respectively. It was assumed that the 
Jun Ando et al.: Changes in the Mandibular Condyle

bone morphological changes appeared in the mandibular angle region because the side shift plate was removed at 16 weeks of age in our long-term experimental group, which may have resulted in the significant difference.

Additionally, regarding the results of the morphological changes in the mandibular bone and condyle observed using $\mu$ $\mathrm{CT}$, the mandibular bone and condylar morphology returned to the same state as that observed at the time of the initiation of the experiment in the ST group after resolving the cause in comparison with that observed in the LT group. Based on the results of the morphological measurements, observation and discussion, the following point is suggested: when the cause of displacement was eliminated during an early stage of growth (4 weeks), the skeletal displacement of the rats' mandibular bone and condyle configuration showed improvement.

We also observed histopathological and immunohistological changes in the ST and LT groups. Growth suppression of the condylar cartilage was considered to occur due to overload until immediately after removing the appliance in both the ST and LT groups. The MMP-1 expression was enhanced in the proliferative, differentiated and hypertrophic layers of the mandibular condylar cartilage on both the ipsilateral and contralateral sides immediately after plate removal (Table 4). Watanabe et al. ${ }^{11)}$ reported that, when malocclusion is caused by side shift plate attachment in rats, the bilateral temporomandibular joints are altered and removal of the cause reduces the production of MMP-1, -8 and -13 and increases the production of type II collagen. In this study, 1 week after plate removal, the MMP-1 expression was significantly decreased on the ipsilateral and contralateral sides (Table 4).

It has been reported that staining of type II collagen is decreased in the superficial layer of cartilage in humans with osteoarthritis of the knee ${ }^{16)}$. Monfort et al. ${ }^{17)}$ stated that mechanical loading of human chondrocytes induces the MMP-1 gene expression and modulates the type II collagen level, thus suggesting that, in the present study, the mandible was displaced leftward, and the excess load induced the production of MMP and promoted the degradation of type II collagen in the ST and LT groups. In addition, the release from leftward displacement induced by inhibition after plate removal may have caused chondrocyte overgrowth, while endochondral ossification may have been caused by an increase in type II collagen and a decrease in the MMP-1 expression.

Regarding the pathological findings, proliferation of the cartilaginous layer was observed between 1 and 2 weeks after removing the appliance in the experimental group. Considering these results and the findings of morphometry, it was speculated that endochondral ossification occurred during this period. Regarding the expressions of VEGF and CTGF positive cells were noted in parts of the proliferative and differentiated layers, particularly in the hypertrophic chondrocyte layer on both the ipsilateral and contralateral sides after plate removal in both groups (Figs. 5, 6: c-f, i-l). Laterality was noted during the period in which the VEGF- and CTGF-positive areas increased, similar to those within the hypertrophic layer in the proliferative period, and this may have been due to the difference in the compression level between the bilateral posterior regions of the mandibular condyle (Tables 2,3).

Hypertrophic chondrocytes induced bone and blood vessels in the surrounding tissue, playing a central role in osteogenesis. Cartilage grows as a result of rapid chondrocyte proliferation and the secretion of cartilage matrix, but the proliferation finally stops and the chondrocytes become hypertrophic cells. Hypertrophic chondrocytes secrete VEGF, promoting osteogenesis and angiogenesis ${ }^{18,19)}$. Tanaka et al. ${ }^{20)}$ previously reported the involvement of VEGF in cartilage remodeling in osteoarthritis.

Hypertrophic chondrocytes express CTGF at a high level, and CTGF promotes the proliferation and differentiation of chondrocytes and osteoblasts in culture ${ }^{21)}$. Kadota et al. ${ }^{22)}$ applied callus distraction to the rat femur, and observed specific CTGF and CTGF mRNA expression in the osteogenic region by immunostaining and in situ hybridization. We observed that the VEGF- and CTGF-stained areas significantly increased on the ipsilateral and contralateral sides from immediately to 2 weeks after plate removal in both the ST and LT groups (Figs. 5, 6: c-f, i-1). These findings suggest that chondrocyte overgrowth occurred on both the ipsilateral and contralateral sides after plate removal and that the expressions of VEGF and CTGF were markedly enhanced in the hypertrophic cartilage, inducing chondrocyte proliferation and differentiation via endochondral ossification. The reduced CTGF- and VEGF-stained areas observed at 4 and 8 weeks after plate removal may have contributed to calcification of the cartilage maturation process and a reduction in cartilage growth.

Upon comparison of the CTGF- and VEGF-stained areas involved in endochondral ossification between the ST and LT groups, it was noted that the areas increased in the ST group compared to those in the LT group, particularly in the period of active proliferation of the hypertrophic layer at 1 to 4 weeks after plate removal. Nakano ${ }^{7)}$ reported that the rat mandible actively grows until 80 days (11-12 weeks) after birth and reaches maturity after 100 days. Together with our present morphometric findings, these findings indicate that the mandibular morphology markedly recovered in the ST group compared to that in the LT group as a result of a "catch-up" phenomenon, wherein the mandible still had time to reach its full growth potential in the animals treated for a shorter period.

On the basis of the results obtained from this study, the following conclusions can be drawn:

(1) Our data suggest that, when there is functional displacement during the growth period, the mandibular condylar cartilage grows and recovers the morphology of the mandibular 
condyle through a reduction of MMP production and increases in VEGF, and CTGF after early removal of the cause of displacement.

(2) When the cause of displacement was eliminated during an early stage of growth (4 weeks), the skeletal displacement of the rats' mandibular bone and condyle configuration showed recovery, but in the rats treated for a longer term (12 weeks) recovery was not observed. This indicates that correction of occlusal abnormalities at an early stage leads to better recovery of the mandibular configuration.

\section{Acknowledgments}

This work was supported by Grants-in-Aid for Scientific Research from the Japan Society for the Promotion of Science (22592297, 23792449 and 23593044). We are grateful to Prof. Noboru Kuboyama for their expert technical advice and kind help with the study.

\section{References}

1. Dijkgraaf Lc, Lambert Gm, Geert B and Robert Sb. Normal cartilage structure, biochemistry and metabolism: A review of the literature. J Oral Maxillofac Surg 53: 924-929, 1995

2. Haskin $\mathrm{Cl}$, Milam Sb and Cameron IL. Pathogenesis of degenerative joint disease in the human temporomandibular joint. Crit Rev Oral Biol Med 6: 248-277, 1995

3. Kamelchuk Ls and Major Pw. Degenerative disease of the temporomandibular joint. J Orofac Pain 9: 168-180, 1995

4. Myers Dr, Barenie Jt, Bell Ra and Williamson Eh. Condylar position of children with functional posterior crossbite: Before and after crossbite correction. Pediatr Dent 2: 190194, 1980

5. Mongini F and Schmid W. Treatment of mandibular asymmetries during growth. A longitudinal study. Eur J Orthod 9: 51-67, 1987

6. Vig PS and Vig KWL. Hybrid appliance: A component approach to dentofacial orthopedics. Am J Orthod Dentofac Orthop 90: 273-285, 1986

7. Nakano H. The histological and morphological changes in the mandibles and masseter muscles in rats with induced mandibular asymmetry. J Jpn Orthod Soc 55: 111-125, 1996

8. Fuentes MA, Opperman LA, Buschang P, Bellinger LL, Carlson DS and Hinton R J. Lateral functional shift of the mandible: part I. effects on condylar cartilage thickness and proliferation. Am J Orthod Dentofacial Orthop 123: 153159, 2003

9. Petrovic AG, Stutzmann JJ and Oudet CL. Control processes in the postnatal growth of the condylar cartilage of the mandible. Ann Arbor Michigan 4: 101-153, 1975
10. Watanabe A, Yamaguchi M, Fujita S and Kasai K. Growth change of rat mandible after release from mandibular side shift. Jpn J Jaw Deform 17: 22-28, 2007

11. Watanabe A, Yamaguchi M, Utunomiya $T$, Yamamoto $H$ and Kasai K. Histopathological changes in collagen and matrix metalloproteinase levels in articular condyle of experimental model rats with jaw deformity. Orthod Craniofac Res 11: 105-118, 2008

12. Maes C, Stockmans I, Moermans K, Van Looveren R, Smets N, Carmeliet P, Bouillon R and Carmeliet G. Soluble VEGF isoforms are essential for establishing epiphyseal vascularization and regulating chondrocyte development and survival. J Clin Invest 113: 188-199, 2004

13. Kameyama Y. Histologic and histometric study of the effect of fluoride on the rat mandibular condyle. J Oral Path 3: 205-216, 1974

14. Prader A, Tanner JM and von Harnack GA. Catch up growth following illness or starvation. J Ped 62: 646-659, 1963

15. Miki T. Studies on the morphogenesis and age changes of the temporo-mandibular jint of normal rat. Bull Stom Kyoto Univ 12: 1-33, 1972

16. Kubokawa T. The Mechanism of articular cartilage degeneration in osteoarthritis. J Jpn Orthop Assoc 68: 415425, 1994

17. Monfort J, Garcia-Giralt N and Lopez-Armada MJ. Decreased metalloproteinase production as a response to mechanical pressure in human cartilage : a mechanism for homeostatic regulation. Arthritis Res Ther 8: R149, 2006

18. Chung, U.I. Essential role of hypertrophic chondrocytes in endochondral bone development. Endocr J 51: 19-24, 2004

19. Kronenberg H.M. Developmental regulation of the growth plate. Nature 423: 332-336, 2003

20. Tanaka E, Aoyama J, Miyauchi M, Takata T, Hanaoka $\mathrm{K}$, Iwabe $\mathrm{T}$ and Tanne K. Vascular endothelial growth factor plays an important autocrine/paracrine role in the progression of osteoarthritis. Histochem Cell Biol 123: 275281, 2005

21. Takigawa M, Nakanishi $\mathrm{T}$ and Shimo T. CTGF, the Most important factor for endochondral ossification. Cell Technology 17: 357-362, 1998

22. Kadota H, Nakanishi T, Asaumi K, Yamaai T, Nakata E, Mitani S, Aoki K, Aiga A, Inoue H and Takigawa M. Expression of connective tissue growth factor/ hypertrophic chondrocyte-specific gene product 24 (CTGF/Hcs24/CCN2) during distraction osteogenesis. J Bone Miner Metab 22: 293-302, 2004 